\title{
Serum tumor markers for screening and early diagnosis of ovarian cancer
}

\author{
Markeri tumorali serici pentru screening-ul şi diagnosticarea precoce a \\ cancerului ovarian

\section{Octavian Gabriel J. Olaru' ${ }^{1}$, Cătălina Monica C. Pena ${ }^{2 *}$}

1. “Carol Davila” University of Medicine and Pharmacy, Department of Obstetrics and Gynecology, "Sf. Ioan” Emergency Hospital, Bucur Maternity - Bucharest

2. "Ana Aslan” National Institute of Gerontology and Geriatrics,

“Biology of Aging” Research Department - Bucharest

\begin{abstract}
Discovery of new biomarkers or panels of biomarkers for early diagnosis of ovarian cancer is one of the great challenges of modern medicine. The use of determination of CA125 (the most commonly used biomarker) was improved by taking into account the dynamic values available in the context of screening. Currently, the highest sensitivity of screening tests performed for early diagnosis of ovarian cancer is $96 \%$ and was obtained using a panel of serum biomarkers composed of apolipoprotein A1, transthyretin, transferrin and CA125. This paper presents different types of serum proteins that are currently studied, and should be proposed as new biomarkers for detecting ovarian cancer. Future research needs to be conducted in order to find a new tumor marker panel that is quantitative, reproducible, and fast and has an even higher sensitivity and specificity for the early detection of ovarian cancer.
\end{abstract}

Keywords: ovarian cancer; early diagnosis; serum biomarkers or serum tumor markers; sensitivity; specificity.

\section{Rezumat}

Descoperirea de noi biomarkeri sau paneluri de biomarkeri pentru diagnosticarea precoce a cancerului ovarian este una dintre marile provocări ale medicinei moderne. Folosirea determinării de CA125 (biomarkerul cel mai des folosit) a fost îmbunătățită luând în considerare valorile în dinamică, disponibile în contextul screening-ului. In prezent, cea mai mare sensibilitate a testelor screening efectuate pentru diagnosticul precoce de cancer ovarian a fost de $96 \%$ şi a fost obținută folosind un panel de biomarkeri serici compus din apolipoproteina A1, transtiretina, transferina şi CA125. Lucrarea de față prezintă diferite tipuri de compuşi proteici din ser, propuşi ca biomarkeri pentru detectarea cancerului ovarian, care sunt studiați în prezent. Studiile viitoare vor trebui conduse în scopul găsirii unui nou panel de markeri tumorali cantitativ, reproductibil, rapid şi cu sensibilitate şi specificitate chiar mai mari decât cele obținute până acum, pentru diagnosticarea precoce a cancerului ovarian.

Cuvinte cheie: cancer ovarian; diagnostic precoce, markeri tumorali serici; sensibilitate; specificitate.

Received: $16^{\text {th }}$ April 2013; Accepted: 25 ${ }^{\text {th }}$ November 2013; Published: $10^{\text {th }}$ December 2013.

*Corresponding author: Cătălina Monica C. Pena, Bucuresti, sector 3, Aleea Coconi nr 15, cod 081331.

Tel: +0727919065, E-mail: penacata@yahoo.com 


\section{Introduction}

Of all gynecologic malignancies, the most lethal is ovarian cancer. The lack of symptoms in the early stages of the disease and the intraabdominal location make early detection and monitoring of the disease, by conventional methods, difficult. More than $70 \%$ of ovarian cancers are diagnosed in the third or fourth stage of the disease when the 5-year survival rate is less then $20 \%$, even with extensive surgery and chemotherapy (1).

It is wide accepted that early diagnosis is the cornerstone of successful treatment of ovarian cancer. Early diagnosis requires new approaches. An attractive direction is the use of serum tumor markers.

These tumor markers are a great variety of substances who have characteristic the fact that are produced by cancer cells and are eliminated into the blood, where they can be detected. At present, for most diseases, they are used for differential diagnosis, monitoring the response to treatment, detecting relapse and predicting prognosis, but not for early detection or as positive diagnostic tests (2). Although the idea of using tumor markers for early detection of cancer is not new, satisfactory results was not yet achieved in this direction.

Currently, the research on serum ovarian cancer markers is underway in many aspects:

- identification of new, more specific, and sensitive ovarian cancer markers;

- testing of available or validation of potential markers in clinical trials;

- improvement and enhancement of technologies who are used to determine and analyze cancer markers;

- finding markers'association in order to maximize the sensitivity and specificity of detection tests (3)

Therefore, the discovery of these markers and finding useful markers'association is one of the main challenges of modern medicine. This article analyzes the different substances studied as serum markers of ovarian cancer at the present.

\section{Screening problems for ovarian cancer}

The ideal marker is a substance secreted only by cancerous cells (and not by normal cells), and should be detectable in a bodily fluid, in constant levels. Additionally, it should be determined by sufficiently noninvasive and inexpensive methods, who can be used in a widespread screening process for the detection of the disease in asymptomatic women.

Considering the known prevalence data for ovarian cancer, tests used for his detection, in the early stage, must have a high sensitivity (proportion of cancers detected by a positive test), as well as an extremely high specificity (proportion of those without cancer identified by a negative test), to attain a positive predictive value (PPV) of at least 10\% (2 - 4).

Currently, for early-stage detection, there are no markers who are fully satisfying. Only a few markers for ovarian cancer have a sufficiently high sensitivity and even among them, most have a very poor specificity. The most limiting factor is the lack of specificity. Many markers are tumor-associated rather than tumor-specific and are elevated in multiple cancers, benign and physiological conditions also (2).

\section{CA-125 (Cancer Antigen 125)}

The most used marker for ovarian cancer is CA-125, which is a cell surface glycoprotein (5). For the moment the determination of the CA-125 in serum is the "gold standard" in ovarian cancer diagnosis. The serum levels of CA-125 increase in advanced ovarian cancer for about $80-90 \%$ of patients. The great disadvantage is that only $50 \%$ or less of the patients in the first stage of the disease have elevated levels (3). In addition, serum levels are also elevated in several nonmalignant conditions, which can lead to false-positive results.

Recently, new studies show that the effectiveness of the use of CA-125 levels could be improved by using the serial values analysis. 
Jacobs et al. made a study of more than 50.000 determinations of serum CA-125, involving 22.000 volunteers who were followed for an average of 8.6 years. They noted that high CA125 levels in women without ovarian cancer had a static, flat or decreasing profile over time, whereas levels associated with malignancy tended to increase (6). Thus the time evolution of CA-125 levels appear to be more important than the value of a single determination.

These data were used to construct a computerized algorithm to estimate a woman's risk of ovarian cancer (OCR). The algorithm increases the sensitivity compared with a single cut-off value of CA-125 because women with normal but rising levels are identified as being at increased risk, while women with elevated levels who are static are classified as low risk (2). Using this method, for a target specificity of $98 \%$, the OCR calculation achieved a sensitivity of $86 \%$ (Table 1) (2).

In 2009, encouraging results using this algorithm have been reported in the UK (United Kingdom) and USA (United States of America). In the ongoing randomized-controlled UK Collaborative Trial of Ovarian Cancer Screening (UKCTOCS), the multimodal screening using the OCR algorithm achieved a sensitivity of $89.5 \%$, specificity of $99.8 \%$, and PPV of $35.1 \%$ for ovarian epithelial cancers detected in an early stage (7).

In a study realised on 3238 subjects, by $\mathrm{Lu}$ et al., OCR algorithm followed by transvaginal scan had a specificity of $99.7 \%$ and PPV of $37.5 \%$ (8).

\section{HE4 (Human Epididymal Protein 4)}

HE4 is a protein that was first found in the epididymal epithelial cells but is also expressed in other epithelial cells. As a marker, HE4 is was proposed for ovarian cancer. Has been shown that levels of the protein HE4, while not elevated in benign gynecologic conditions, is elevated in epithelial ovarian cancers (EOC), the most common type of ovarian cancer.
In 2009, in the USA, HE4 was approved for monitoring women who had been diagnosed with epithelial ovarian cancer, for indications similar to those of CA-125.

\section{Combination of the determination of CA- 125 and HE4}

Even initially, the combination of the determination of CA-125 with HE4 seemed to be promising by increasing the sensitivity and the specificity in detecting ovarian cancer, more recently, by analyzing the results of other studies, some researchers concluded that there is no benefit in clinical settings $(2,6,9$ - 11).

In 2011, in the USA was approved the marketing of the HE4 Test along with the CA125 test. These tests were combined in the Risk of Ovarian Malignancy Algorithm, called ROMA, to determine the likelihood of finding malignancy at surgery in women who have an adnexal mass. The diagnostic performance of ROMA was advocated for the first time by Moore et al. (12) who sustained that CA-125 combined with HE4 reveals the highest sensitivity and specificity among nine markers studied.

The Risk of Ovarian Malignancy Algorithm (ROMA) combines the result of the determinations of CA-125 and HE4, by taking in consideration of the premenopausal or the menopausal status, and converting them into a numerical score. ROMA is interpreted in conjunction with an independent clinical and radiological assessment. The aim is to aid the assessing for premenopausal or postmenopausal woman, who presents an ovarian adnexal mass, and to estabilish if there is a high or low likelihood of finding malignancy at surgery. The ROMA test is indicated for women with age over 18, who have an ovarian pelvic mass for which surgery is planned and are not yet referred to an oncologist. In the Important Safety Information, extracted from the prescribing information provided by the company that produces and promotes test, Fujirebio Diagnostics 
Table 1. Sensitivity and specificity of serum biomarker panels for detection of ovarian cancer

\begin{tabular}{lclcccc}
\hline Autor & Year & Panel/biomarkers & No subjects & Sensitivity & Specificity & Ref. \\
\hline Jacobs & 1999 & OCR algorithm & 22,000 & $86 \%$ & $98 \%$ & 8 \\
Zhang & 2004 & Apo A1, TTR, H4, CA 125 & 503 & $74 \%$ & $97 \%$ & 10 \\
Mor & 2005 & Prolactin, OPN, leptin, IGF-II & 206 & $95 \%$ & $95 \%$ & 11 \\
Visintin & 2008 & Leptin, prolactin, OPN, IGF-II, & 518 & $95.3 \%$ & $99.4 \%$ & 12 \\
& MIF, CA125 & & & & \\
Nosov & 2009 & Apo A1, TTR, TF, CA 125 & 358 & $96 \%$ & $96 \%$ & 13 \\
\hline
\end{tabular}

Inc., it shows that ROMA "should not be used without an independent clinical/ radiological evaluation and is not intended to be a screening test or to determine whether a patient should proceed to surgery" and follows "Incorrect use of ROMA carries the risk of unnecessary testing, surgery, and/or delayed diagnosis" (13).

\section{Additional markers}

To date, no marker or algorithm has met the criteria for early diagnosis of ovarian cancer. There is a great need for additional markers to improve sensitivity of detection with retained or better specificity. Efforts have been made to improve the tests' performance by discovering and combining new markers, which continue to be evaluated. Current studies show that combining other several markers, which could be added to the determination of CA-125, could improve the sensitivity of ovarian cancer detection (2).

In a five-center case-control study by Zhang et al. from 2004, serum proteomic expressions were analyzed for 153 patients with invasive epithelial ovarian cancer, 42 with other ovarian cancers, 166 with benign pelvic masses and 142 healthy women. Data from patients with early stage ovarian cancer and healthy women were analyzed independently and the results cross-validated in order to discover potential markers. After protein identification, three of them seems to be important: apolipoprotein A1 (down-regulated in cancer), a truncated form of transthyretin (TTR) (down-regulated), a cleavage fragment of inter-alpha-trypsin inhibitor heavy chain $\mathrm{H} 4$ (up-regulated).

Using a model that combines these three markers and CA-125 in order to detect early stage invasive epithelial ovarian cancer in healthy controls the sensitivity obtained was $74 \%$, higher than that of CA-125 alone. When compared at a fixed sensitivity of $83 \%$, the specificity of the model (94\%) was significantly better than that of CA125 alone (52\%) (14 - 16). These markers demonstrated potential to improve the detection of early stage ovarian cancer (Table 1).

In a study by Mor et al. from 2005, the levels of 169 serum proteins were analyzed from 18 patients with untreated epithelial ovarian cancer (EOC) and 28 healthy age-matched controls, using receiver operating characteristic (ROC) curve with immunoassay microarray (10). Of these 169 proteins only leptin, prolactin (PRL), osteopontin (OPT) and insulin-like growth factorII (IGF-II) had significant differences between disease-free and cancer patients. Then the combination of these four markers was tested in a blinded cross-validation study on 206 serum samples, 106 coming from healthy subjects and 100 from patients with ovarian cancer. Both PRL and OPT are significantly elevated in the serum of patients with EOC, whereas leptin and IGF-II levels are reduced. The final results of the test have a sensitivity of $95 \%$, a specificity of $95 \%$, a PPV of $95 \%$, and a negative predictive value (NPV) of 94\% (10) (Table 1).

Another assay that used a panel of six serum markers, leptin, PRL, OPT, IGF-II, 
macrophage inhibitory factor (MIF), and CA125, was studied on 362 healthy controls and 156 patients with ovarian cancer, including 13 stage I cases, yielded a $95.3 \%$ sensitivity and $99.4 \%$ specificity $(3,8)$.

A several studies published in 2008 by Hellstrom and al., were focused on the role played by soluble mesothelin-related proteins (SMRP) and HE4 in ovarian cancer, but also other markers were taken into account. Mesothelin is a protein that was discovered in mesothelial cells but her physiological role is not well known. It is presumed that mesothelin may be involved in cell adhesion and it was found that she is overexpressed in several human tumors, including mesothelioma, ovarian and pancreatic adenocarcinoma. The studies reveal that mesothelin has a relatively high specificity for ovarian cancer. In the cases of ovarian cancer with little or no detectable CA-125 expression, mesothelin and HE4 stood out as the most promising markers $(17,18)$.

The objectives of a study from 2009 by Nosov et al., were to test whether the panel of serum biomarkers Apo A1, TTR, and TF (transferrin) either with or without CA-125 can detect early-stage ovarian cancer and even find differences between different histological forms (19). First, sensitivity, specificity, and OCR were determined for Apo A1, TTR and TF panel. In that case sensitivity and specificity for detection of earlystage ovarian cancer were $86 \%$ and grow to $94 \%$ for late-stages ovarian cancer. Second, to assess the contribution of CA-125 to the biomarker panel, a separate analysis was performed including determination of CA-125. Combining the results of determinations for all the four biomarkers brought the sensitivity and specificity to $96 \%$. The early-stage ovarian cancer group was further subdivided into histological categories. Each histological subtype was analyzed separately to determine the sensitivity and specificity of the biomarker panel for detection of different subtypes of ovarian carcinoma, excluding mucinous subtype. Identification of endometrioid tumors was associated with a sensitivity and specificity of $98 \%$. To date, $96 \%$ is the highest sensitivity recorded for the early detection of ovarian cancer. However, because the low prevalence of ovarian cancer, a sensitivity and specificity of $96 \%$ would only detect 1 case of cancer per 100 oophorectomies done for positive screens due to the high false-positive rate (19). Future research needs to be conducted to find a tumor marker panel that has an even higher sensitivity and specificity (Table 1).

The research of Dieplinger $\mathrm{H}$. et al., published in 2009, about the role of afamin, led to the discovery that this substance may indirectly help to detect and follow the evolution of ovarian cancer. Afamin, which was discovered by the same group, is a vitamin E-binding glycoprotein, a member of the albumin family, synthesized by the liver, and secreted in plasma. It can also be found in substantial quantities in the extravascular fluid. Afamin concentrations in serum decreased in pre-operative patients diagnosed with ovarian cancer and rose again after tumor removal and chemotherapy. In conclusion, afamin, possibly in combination with other already established markers, may serve in a panel for early detection of ovarian cancer (20).

In a study from 2011 Zhu C.S. and al. started from the idea that until then, serum samples, who was used, collected at the time of diagnosis generally included a high proportion of cases with advanced stage diseases (21). Thus, their utility for screening, which requires detection during an asymptomatic phase, cannot be determined. Therefore, this study provided the first example of a systematic approach to a marker validation using prediagnosis specimens. Indeed, it is possible that markers discovered in diagnostic samples can have significantly different values only when the tumor increases in size or is clinically observable. Such markers may have little value for early diagnosis (19).

Using a nested case-control design, 28 markers were measured laboratory-blinded in 118 serum samples obtained before cancer diagnosis and 951 serum samples from matched 
Table 2. Summary of five predictive models of biomarker panels in a study from Zhum et al. (2011)

\begin{tabular}{lccccc}
\hline Panel & A & B & C & D & E \\
\hline Markers & CA125 & B7-H4 & CA125 & APOA1 & CA125 \\
& IGF-II & CA125 & HE4 & B2M & CA72-4 \\
& Leptin & CA15-3 & IGFBPII & CA125 & EGFR \\
& MIF & CA72-4 & Mesothelin & CTAPIII & Eotaxin \\
& OPN & HE4 & MMP-7 & TTR & HE4 \\
& Prolactin & & SPLI & & MMP-3 \\
& & & SPON2 & & Prolactin \\
& & & & sVCAM-1 \\
\hline
\end{tabular}

SPLI - secretory leukocyte protease inhibitor, SPON2 - spondin2, B2M - beta-2-microglobulin.

controls (21). They were grouped into five model panels (Table 2), each containing six to eight biomarkers.

Results showed that only one model showed comparable performance to CA-125, with sensitivity and specificity at $69.2 \%$ and $96.6 \%$ respectively (ROC 0.892). Remaining models had poorer performance than CA-125 alone. Even a model derived from all 28 markers failed to show improvement over CA-125. Also in the study conclusions it warns that, it may be possible that indicator panels found in samples from patients with confirmed diagnosis do not have the same importance in prediagnostic samples $(21,22)$.

\section{Discussions}

The ability to reduce cancer mortality through population screening is widely accepted and consequently, extensive efforts are underway to explore this possbility for all forms of cancer. Screening methods depend on the characteristics of the disease to be diagnosed. For the ovarian cancer direct examination or even imaging methods can not give satisfactory results, in early stage, due to his localization. Idea of using serum tumor markers for this purpose seems attractive although there are significant barriers that must be overcome.

In the last years, promising markers for ovarian cancer diagnosis were discovered and studied. Of these proteins only mesothelin, OPT and HE4 have been selected by the SPORE (Specialized Program of Research Excellence) committee for their high level of sensitivity and specificity in differentiating EOC from normal ovarian epithelium (17) but many more awaits validation in clinical trials.

Other substances, such as TF, are acute phase proteins and have been associated with systemic inflammation as well as other non-cancerous conditions $(19,23)$. Afamin could add independent diagnostic information to CA-125 and other markers but it is not suitable as single diagnostic marker for ovarian cancer (20).

However, even they are not all cancer-specific markers or all produced directly by the ovarian cancer cells, they can be useful for differentiating the serum samples from pacients with cancer from samples of patients with benign conditions or healthy.

\section{Conclusions}

To date there is no adequate screening test for the early detection of ovarian cancer.

Serum CA-125 remains the best marker for ovarian cancer in single determination, with sensitivity of $86 \%$ in cases where blood was drawn within 6 months from diagnosis.

The second best marker is HE4, with sensitivity of $73 \%$.

The biomarker panel, which includes Apo A1, TTR, TF, and CA-125 has the highest 
sensitivity for early-stage ovarian cancer reported to date, but it needs to be higher in order to validate this panel of biomarkers as an effective screening tool for ovarian cancer.

Mesothelin has a relatively high specificity for ovarian cancer and for the cases of ovarian cancer where is no detectable or little CA-125 expression, is a promising marker who can increase the sensitivity and the specificity of an biomarker panel test if included.

Although there are substances that do not comply with the classic definition of tumor markers (like afamin) can be used in combination with authentic markers within panels and can be useful for early detection of ovarian cancer.

The growing understanding of ovarian cancer etiology and subtypes, increasing attention in design of biomarker studies, and use of new technologies on carefully selected pre-diagnostic sample sets, give hope for identification of novel biomarkers.

The identification of more sensitive and specific markers or marker panels for the early detection of ovarian cancer would be immediately beneficial because the majority of patients with ovarian carcinomas are still diagnosed in the late stages.

In the future, it will still be crucial to further develop panels of biomarkers not only for early detection but also for treatment guidance of ovarian cancer and detecting recurrence.

\section{Conflicts of interest}

The authors report no conflict of interest. We declare that we are not involved in any conflicts of interest and we have no source of funding.

\author{
Abbreviations \\ Apo A1 - apolipoprotein \\ TTR - transthyretin \\ PPV - positive predictive value \\ NPV - negative predictive value \\ OCR - risk of ovarian cancer \\ EOC - epithelial ovarian cancer \\ ROC - Receiver operating characteristic curve
}

OPT - osteopontin

IGF-II - insulin-like growth factor-II

MIF - macrophage inhibitory factor

$\mathrm{TF}$ - transferrin

\section{References}

1. Guenther J, Stiles A, Champion JD The lived experience of ovarian cancer: a phenomenological approach. J Am Acad Nurse Pract. 2012; 24(10): 595-603.

2. Gentry-Maharaj A, Menon U Screening for ovarian cancer in the general population. Best Practice \& Research Clinical Obstetrics \& Gynaecology. 2012; 26: 243-256.

3. Zhang B, Cai FF, Zhong XY An overview of biomarkers for the ovarian cancer diagnosis. European Journal of Obstetrics and Gynecology. 2011; 158(2): 119-123.

4. Zang B, Barekati Z, Kohler C, Radpour R, Asadollahi $\mathrm{R}$, Holzgreve $\mathrm{W}$ et al. Proteomics and Biomarkers for Ovarian Cancer Diagnosis, Annals of Clinical \& Laboratory Science. 2010; 40(3): 218-225.

5. O'Malley D, Azodi M, Makkenchery A, Tangir J, McAlpine J, Kelly M. Serum protein markers for early detection on ovarian cancer. PNAS 2005; 102: 7677-82.

6. Jacobs IJ, Skates SJ, MacDonald N, Menon V, Rosenthal AN, Davies AP et al. Screening for ovarian cancer: a pilot randomised controlled trial. Lancet 1999; 353: 1207 1210.

7. Moore R G, Maclaughlan S Current clinical use of biomarkers for ovarian cancer. Current opinion in oncology. 09/2010; 22(5):492-7.

8. Lu KH, Skates S, Bevers TB, Newland W, Moore RG, Leeds L et al. A prospective US ovarian cancer screening study using the risk of ovarian cancer algorithm (ROCA). J Clin Oncol. 2010; 28:[suppl; abstract 5003]

9. Moore RG, McMeekin DS, Brown AK, DiSilvestro P, Miller MC, Allard WJ et al. A novel multiple marker bioassay utilizing HE4 and CA125 for the prediction of ovarian cancer in patients with a pelvic mass. Gynecologic Oncology. 2009;112: 40-46.

10. Mor G, Visintin I, Lai Y, Zhao H, Schwartz P, Rutherford T et al. Serum protein markers for early detection of ovarian cancer. Proc. Natl. Acad. Sci. U.S.A..May 2005; 102(21): 7677-7682.

11. Jacob F, Meier M, Caduff R, Goldstein D, Pochechueva T, Hacker $\mathrm{N}$ et al. No benefit from combining HE4 and CA125 as ovarian tumor markers in a clinical setting. Gynecol Oncol. 2011; 121: 487-491.

12. Moore RG, Miller C, DiSilvestro P, et al. Evaluation of the diagnostic accuracy of the risk of ovarian malignancy algorithm in women with a pelvic mass. Obstet Gynecol. 2011; 118(2):280-288.

13. ROMATM (HE4 EIA + ARCHITECT CA 125 IITM) prescribing information. Fujirebio Diagnostics, Inc., 2011. 14. Zhang Z, Bast RC, Yu Y, Li J, Sokoll LJ, Rai AJ et al. Three biomarkers identified from serum proteomic 
analysis for the detection of early stage ovarian cancer. Cancer Res. Aug 2004; 64(16): 5882-90.

15. Zhang Z, Chan DW Cancer proteomics: In Pursuit of "True"Biomarker Discovery. Cancer Epidemiol Biomarkers Prev. 2005; 14: 2283-2286.

16. Song J, Daniel W, "Cancer proteomics". Clinical proteomics. 12/2006; (1542-6416), 2(3): 129.

17. Hellstrom I, Hellstrom KE SMRP and HE4 as Biomarkers for Ovarian Carcinoma When Used Alone and in Combination with CA125 and/or Each Other. Advances in Experimental Medicine and Biology. 2008;622:15-21.

18. Hellstrom I, Friedman E, Verch T, Yang Y, Korach J, Jaffar $\mathbf{J}$ et al. Antimesothelin antibodies and circulating mesothelin relate to the clinical state in ovarian cancer patients. Cancer Epidemiol Biomarkers Prev. 2008;17:15206.

19. Nosov V, Su F, Amneus M, Birrer M, Robins T, Kotlerman $\mathbf{J}$ et al. Validation of serum biomarkers for detection of early-stage ovarian cancer. Am J Obstet Gynecol.
2009; 200:639

20. Dieplinger H, Ankerst DP, Burges A, Lenhard M, Lingenhel A, Fineder L et al. Afamin and apolipoprotein A-IV: novel protein markers for ovarian cancer. Cancer Epidemiol Biomarkers Prev. 2009; 18: 1127-1133.

21. Zhu CS, Pinsky PF, Cramer DW, Ransohoff DF, Hartge P, Pfeiffer RM et al. A Framework for evaluating biomarkers for early detection: validation of biomarker panels for ovarian cancer. Cancer Prev Res. 2011; 4: 375383.

22. Bast RC, Badgwell D, Lu Z, Marquez R, Rosen D, Liu $\mathbf{J}$ et al. New tumor markers: CA125 beyound. Int $\mathbf{J}$ Gynecol Cancer. 2005; 15: 274-281.

23. Moore RG, Brown AK, Miller MC, Skates S, Allard WJ, Verch $\mathrm{T}$ et al. The use of multiple novel tumor biomarkers for the detection of ovarian carcinoma in patients with a pelvic mass. Gynecol Oncol. 2008; 108: 402408. 